\title{
Educación artística, intercreatividad y postdigitalidad: reflexión crítica sobre sus relaciones y procesos en tiempos del covid-19
} Arts education, intercreativity and postdigitality: a critical reflection in times of covid-19

\author{
Julia MAÑERO. Universidad de Sevilla (España). juliamanero@us.es
}

Resumen: La presente reflexión toma como punto de partida la situación pandémica que nos atraviesa para dilucidar otras formas posibles de hacer y comprender la educación artística. La situación de crisis sanitaria global ha visibilizado la importancia de las artes y la cultura en una sociedad donde en gran medida las prácticas educativas (artísticas) siguen careciendo de imaginación, creatividad y valores democráticos. Se antoja necesario un breve análisis de la importancia de las artes y su educación, así como el nuevo marco social en el que se insertan: la postdigitalidad. La presencia de las tecnologías y dispositivos digitales -aún más si cabe durante el periodo de pandemia- ha evidenciado una hibridez entre los límites físicos y digitales, dos contextos íntimamente interrelacionados. La educación, al igual que las artes y su práctica educativa, se nutre de estos espacios y modos de hacer y es necesaria una mirada (crítica) hacia las posibilidades y retos que presentan. Así, una de las estrategias pedagógicas que surge en este análisis es el concepto de intercreatividad. Un discurso que se aproxima a los procesos creativos colectivos desde una concepción educativa crítica reconoce la concepción artística como un poderoso recurso pedagógico en el aula. La intercreatividad se posiciona como una práctica artística y una estrategia educativa a través de la creación colectiva que focaliza su atención en la mejora y en el cambio social.

Palabras clave: artes, covid-19, educación artística, intercreatividad, pedagogía crítica, postdigitalidad.

\begin{abstract}
The following reflection considers as a starting point the pandemic that is currently affecting us in order to clarify other possible ways of doing and understanding arts education. The global sanitary crisis has made visible the importance of arts and culture in a society where educational (artistic) practices are still mostly lacking in imagination, creativity and democratic values. A brief analysis of the importance of the arts and their education is necessary, as well as the new social framework in which they are inserted: postdigitality. The presence of digital technologies and devices -even more so during the pandemic period- has shown a hybridisation between physical and digital
\end{abstract}


boundaries, two closely interrelated contexts. Education, like the arts and its educational practice, is nourished by these spaces and ways of doing things, and a (critical) look at the possibilities and challenges they present is necessary. Thus, one of the pedagogical strategies that emerges in this analysis is the concept of intercreativity. A discourse that approaches collective creative processes from a critical educational conception recognises the artistic conception as a powerful pedagogical resource in the classroom. Intercreativity is positioned as an artistic practice and an educational strategy through collective creation that focuses its attention on improvement and social change.

Keywords: arts, arts education, covid-19, critical pedagogy, intercreativity, postdigital.

\section{Introducción}

Cumplido más de un año desde la proclamación de una crisis sanitaria sin precedentes y estados de alarma globalizados, las debilidades del actual sistema económico-social siguen manifestándose y afectando a nuestra estructura social en todas sus vertientes. En este periodo pandémico, el virus denominado COVID-19 -conocido comúnmente como coronavirus- ha visibilizado muchas de las limitaciones de la sociedad actual, reticencias que permanecían en un segundo plano y que han precipitado el colapso del mercado y, por tanto, del sistema capitalista. La dudosa normalidad, antes del primer indicio del virus, estaba marcada por ritmos de vida acelerados y líquidos (Bauman, 2006). Una sociedad hiperconectada, pero al mismo tiempo dominada por la individualidad y la competitividad. Un modelo de sociedad que mantiene causas diversas, y para su dilucidación podemos hallar algunas respuestas dentro del enfoque del sistema educativo que lo sustenta.

En términos globales, la estructura educativa sigue apoyada en patrones reproductivos y bancarios, y continúa perdiendo oportunidades para cambiar ese enfoque. Desde el área de conocimiento que nos ocupa, la educación artística, un ejemplo evidente son la mayoría de las legislaciones educativas vigentes que menosprecian y desvalorizan el potencial de las artes en el desarrollo humano. Si nos remitimos al contexto español, la aprobación de una nueva ley educativa en diciembre de 2020: Ley Orgánica 3/2020, de 29 de diciembre, por la que se modifica la Ley Orgánica 2/2006, de 3 de mayo, de Educación es un claro ejemplo. Dicha ley volvió a relegar las artes y la cultura a un segundo plano a pesar de la presencia de dinámicas activistas colectivas ${ }^{1}$, críticas al respecto en los medios de comunicación y aportando razonamientos fundamentados sobre la cultura y las artes como conocimientos indispensables en un sistema educativo (crítico) de calidad. En definitiva, y de manera generalizada, se siguen perpetuando ciertas decisiones educativas que se materializan en oportunidades perdidas para potenciar las artes como mecanismo de cambio social.

A pesar de ello, desde los inicios de esta crisis sanitaria los procesos creativos, culturales y artísticos han servido de nexo común entre los diferentes territorios que están batallando

1 Un ejemplo sería la Plataforma \#EducaciónNoSinArtes cuyas enmiendas al proyecto de ley, junto con el decálogo para su mejora, obtuvieron repercusión mediática y sensibilización social. Puede consultarse en https://educacionnosinartes.wordpress.com. 
contra una pandemia globalizada. Es necesario matizar que no significa que estos procesos inicialmente no estuvieran dotados de relevancia social, sino que esta notabilidad se ha visto acentuada por una crisis sanitaria sin precedentes. Un amplio sector de la sociedad ha trabajado colectivamente, revisando las formas de ser y estar en comunidad, y reconociendo la importancia de lo social como punto de encuentro con nosotras y nosotros mismos y con los demás; acciones culturales, y dinámicas artísticas colectivas desde ventanas, balcones y en espacios digitales (Jandrić et al., 2020).

La acción solidaria y colectiva ha sido generalizada, como afirma el filósofo alemán Gabriel Markus (2020), es necesaria una educación crítica y ética que nos haga deliberar y replantearnos el orden mundial competitivo, fomentando lo común, la colaboración. Es en este momento cuando hay que tomar la decisión de cómo educar, es el punto de inflexión para decidir entre seguir reproduciendo la educación bancaria e instructiva $o$, siguiendo a Noam Chomsky, educar para que las niñas y niños comprendan el mundo y su funcionamiento (AprendemosJuntos, 2020). Como indica el prestigioso lingüista, es importante que exploren el mundo, que se estimule su propio instinto creativo y la imaginación. Esa creatividad paulatinamente mermada a lo largo de las etapas escolares, una creatividad mitigada y minusvalorada (Giroux, 2015; Robinson, 2009), es vital para el desarrollo humano, para el avance hacia una sociedad crítica y reflexiva. La creatividad forma parte de lo que el pedagogo crítico Henry Giroux denomina "pensamiento peligroso". La creatividad, movida por la imaginación, es parte de los procesos artísticos y la capacidad de estos para vislumbrar otras formas posibles de ser y estar, tan necesarias después de esta pandemia virulenta fruto de nuestra sociedad.

\section{La necesidad de una educación artística (crítica)}

La importancia de las artes como un vehículo para la transformación y la emancipación social deviene de su propio significado. Atendiendo al sentido posmoderno de nuestra contemporaneidad el término arte se comprende como una producción cultural que refleja las características propias e intrínsecas de cada cultura (Efland et al., 2003). A pesar de equipararse cultura y arte a una misma manifestación, igualmente relevante, sigue existiendo una perspectiva predominante en la que se equipara a las prácticas artísticas a meras actividades de esparcimiento y reciben una falta de desconsideración en términos sociales (Vaquero y Gómez del Águila, 2018). Por ello, es necesario rescatar la importancia de las artes en la sociedad, entendiéndolas como instituciones sociales que influyen y son influidas por las culturas y subculturas desde las que parten (Chalmers, 2003). Al ser las artes una representación simbólica de las características definitorias de una sociedad o colectivo social, funcionan como un lenguaje, como vehículos de comunicación. Por este motivo el conocimiento de una sociedad y su cultura será sesgado y parcial si no se conocen sus manifestaciones artísticas (Barbosa, 2012).

Por consiguiente, los conceptos arte y sociedad son inseparables, se retroalimentan y ambos proceden del origen de los seres humanos con el medio natural (Read, 1969). De estas premisas se deduce que el arte y las manifestaciones creativas, ayudan a conocer el mundo y nuestra 
posición en él. Es decir, la realidad y nuestra propia contemplación de ella. A través del arte, el ser humano intenta comprender e intervenir en el mundo que observa, y al desarrollar un ejercicio de recreación de lo observado, lo transforma (López Fernández-Cao, 2008). Así, el arte se convierte en un proceso abierto a la reinterpretación, y es en ese momento de libertad en el que el arte se conforma como un agente liberador. Si por el contrario, se marginan manifestaciones artísticas en concreto o las prácticas artísticas se separan de la sociedad, el arte funcionaría como una forma de opresión (Reitz, 2000 en McLaren y Jaramillo, 2010). Una de esas formas de opresión la explica Herbert Read (1969) en la relación que establece entre arte y alienación social. En su tesis arroja algunas conclusiones sobre la necesidad de fomentar y educar en la capacidad de ver y manipular el contexto natural e inmediato. De esta manera se evitaría lo que se denomina una atrofia de la sensibilidad que, unida a una progresiva automatización de los procesos, resultan en seres humanos anestesiados e indiferentes, que buscan distracciones visuales que les alienten a encontrar algún tipo de sentimiento y excitación.

En su afán por no sucumbir a la alienación social, y en la búsqueda del arte como un medio de emancipación social, Read (1969) propone una transformación social a través de lo que denomina una educación por el arte. Una educación por el arte no es anticientífica, ni mecánica, no es una actividad de esparcimiento pasiva. Al contrario, la educación por el arte es revolucionaria, es crítica e imaginativa. La teoría de Herbert Read (1969) se encuentra íntimamente relacionada con lo que hoy conocemos como Educación Artística. Este término, genera controversia ya que, como señala Barragán (2005), existe una confusión en la denominación del área artística, también conocida como educación estética, artes plásticas o cultura visual entre otras denominaciones a día de hoy vigentes, y empleadas según los contenidos u objetivos que se pretenden destacar de dicha formación en y para las artes. Estando de acuerdo con el pensamiento de José María Barragán (2005), en lo que se refiere al término de educación artística como el menos excluyente, a lo largo de estas líneas será el empleado para designar lo que se conoce como un rechazo a las artes como únicamente un modo expresivo, productivo o instrumental. Al contrario, se defiende la educación artística enmarcada en una forma de conocimiento humano con una estructura propia. De esta manera el potencia de las artes, y por ende de la educación artística, reside en una educación que, apuesta por la construcción de significados, con un interés por las manifestaciones culturales (artísticas o no), y promueve la apreciación y la creación de artefactos (audio)visuales tan presentes en nuestra cultura posmoderna y postdigital.

Planteando la importancia y la relevancia de las artes en general y de la educación artística en particular, se visualiza una incoherencia con la realidad educativa que mayoritariamente implementa la educación artística como un desarrollo de destrezas o habilidades manuales (Arañó, 2003) o como una evolución de la autoexpresión creativa. Paralelamente, y en gran medida, es considerada un mero pasatiempo o actividad lúdica (Chalmers, 2003) y sufre un déficit de formación del profesorado encargado de la enseñanza de las artes (PalaciosGarrido, 1999). De manera generalizada, muchos países recrean en sus currículums la idea de que las artes y su enseñanza es prescindible para el alumnado, priorizando otras materias de carácter lógico-matemático (Escaño y Marfil-Carmona, 2020). 
Expuestas de manera sintética algunas premisas, se antoja necesario, como sentencia Mesias-Lema (2019), otros modos de hacer, entender e investigar en educación artística. Las prácticas artísticas, en palabras del autor, manifiestan la posibilidad de canalizar y transformar la realidad social, no de una manera radical, sino posibilitando un encuentro de diferentes miradas a través de la sensibilidad estética que otorgan las experiencias artísticas bajo una perspectiva crítica y cognitiva. La experiencia artística, siguiendo a Dewey (2008), se encuentra íntimamente relacionada a los modos de hacer, una sucesión de actos no caprichosos entre sí, sino el resultado de una percepción profunda al estar el sujeto involucrado emocionalmente. El prestigioso filósofo y psicólogo entiende que, para percibir de manera eficiente, hay que sumergirse en la naturaleza del acto, con el fin de asimilar lo que sucede. Es un acto activo y no pasivo, donde se genera una experiencia propia, una recreación que implica más allá de una experiencia estética. Es una interacción estrecha con lo percibido a través de los sentidos, y por tanto ninguna experiencia artística se sucede de manera idéntica en la humanidad.

En los procesos artísticos se desarrollan tantos procesos cognitivos como emocionales, y por ello estos no difieren profundamente del método científico tradicional. A través de la creación se proyecta una realidad propia y se (re)imaginan posibilidades comprobando al mismo tiempo, su eficacia mediante un control y evaluación. Por ello, la experiencia artística posibilita la experiencia con nuestro propio mundo, recreándolo, siendo constantemente elaborado. Siguiendo esta línea de pensamiento, en el proceso creador artístico se favorece una desarrollo del ser humano, al considerar el arte, la estética y la lúdica como vehículos para promover la crítica, la reflexión y el análisis. Mediante el desarrollo de la imaginación, técnicas y sensibilidad se generan experiencias estéticas, contrapuestas a lo que se conoce experiencias anestésicas que adormecen las sensaciones (Eisner, 2002). Igualmente, a través de las artes se desarrolla la percepción y la creatividad (Barbosa, 2012), se fomenta la resolución de conflictos y la tolerancia de miradas, de percepciones del mundo y sus construcciones posibles (Maeso-Broncano, 2017). El objetivo de la educación artística multicultural, según indica Chalmers (2003), es comprender y valorar las diferentes culturas a través de las manifestaciones artísticas propias a cada una de ellas. De esa manera se exploran temas comunes y distintivos entre culturas, erradicando la tendencia occidental de valorar el arte en función de la complejidad de la cultura en la que se inserta. Como indica Anderson (2003), el sentido de comunidad que implica el arte debe ser global más que tribal; la búsqueda de creencias compartidas y el abarcamiento de múltiples narrativas y perspectivas culturales. Como conclusión a todas las aportaciones y valores que se desprenden de las enseñanzas artísticas, podríamos afirmar que, en palabras de Eisner, la educación artística, cuando es eficaz, potencia ciertas actitudes y un apetito estético y sensible que fomenta un transferencia del conocimiento fuera de las instituciones educativas (Eisner, 2002).

\section{Desarrollando una mirada postdigital de la educación artística}

Como apuntábamos al comienzo de esta reflexión, el papel de las artes y los procesos creativos e intersubjetivos se han reforzado durante esta etapa pandémica. El concepto de postdigitalidad ha sido más latente y hemos tomado conciencia de cómo la tecnología y los dispositivos digitales son parte de nuestra realidad, la cual podría ser catalogada como una 
realidad híbrida e hiperconectada. Los contextos físicos y digitales se retroalimentan, se solapan y tienen lugar de manera simultánea, produciendo un cambio de paradigma en las prácticas educativas (Jandrić et al., 2018, 2020). Lo interesante, en términos pedagógicos, es que una concepción postdigital de la práctica educativa no se reduce a una mera alfabetización instrumental, sino al desarrollo de una mirada crítica hacia la postdigitalidad de nuestra existencia.

Estando la educación artística atravesada por el medio más determinante e influyente hoy día (Internet), el currículo posmoderno debe incorporar los cambios culturales sustentados en la Web, esas nuevas formas de representaciones que harán del propio currículo un collage de la experiencia contemporánea (Efland et al., 2003). Si partimos de Internet como el motor de cambio principal en la actualidad, no se trataría como se ha venido haciendo en multitud de ocasiones, de introducir Internet en el currículo como un mero instrumento. Internet desde la perspectiva de la investigadora y profesora Remedios Zafra ha posibilitado un cambio:

Hoy cambian las relaciones con las personas, cambia la vivencia de los espacios; la visión y la memoria son otras bajo el filtro de la interfaz y de una, tan fascinante como perversa, hipervisibilidad que transforma el acceso y la percepción de las cosas; el mundo después de Internet no es ya lo que era, ni la relación de las personas con las prácticas de conocimiento puede ser tratada igual. (Zafra, 2015, p.13)

Al igual que las manifestaciones artísticas se retroalimentan de estos nuevos espacios y sus posibilidades, desde el punto de vista pedagógico artístico se visibilizan varias potencialidades. Internet es el medio educativo multimedia óptimo hasta la actualidad y la educación artística, al igual que otras áreas de conocimiento, se interrelacionan con él (Escaño, 2009). Al igual que las relaciones sociales — como indicaba Zafra (2015) - se modifican, también lo hacen los contextos educativos. Así lo indica el profesor de educación artística Carlos Escaño, cuando establece una comparativa entre el hipocontexto (contexto original, en nuestro caso educativo) y el hipercontexto (contexto educativo ampliado y modificado en Internet). Una educación artística desarrollada en y para el hipercontexto debe poseer trascendencia educativa, es decir una pedagogía de base, una pedagogía colaborativa, democrática, y ser dotada de un conocimiento para la representación iconográfica.

Al hablar de la importancia de la educación artística y su carácter postdigital, no debemos obviar el espíritu participativo, constructor y en definitiva político que deben promover las artes (Escaño, 2015), hecho que repercute en la labor docente y la concepción de esta (Jiménez, 2020). Como señalan McLaren y Jaramillo (2010), reivindicando el discurso de Theodor W. Adorno, las artes poseen un potencial crítico, una función cognitiva y crítica a la hora de relevar las contradicciones que establece el sistema capitalista. La pedagogía crítica vinculada a las artes ofrece una alternativa, una reconstrucción social que interpela no únicamente a las personas más vulnerables o más socialmente marginadas. En definitiva, la finalidad de la pedagogía crítica artística es la creación de nuevos mundos culturales y sociales.

Este cambio social se persigue desde una educación artística comprometida democratizadora. Aquella que se adapta al contexto, relegándose a una instrucción de pautas y habilidades para competir en el sistema implantado, es una educación artística que fracasa en su objetivo 
principal: proveer de un pensamiento crítico y creativo favorecedores de otros mundos posibles (Escaño y Marfil-Carmona, 2020). La pedagogía contranarrativa o pedagogía crítica artística, se diferencia de la pedagogía crítica precisamente por esta propuesta de creación cultural y social. La pedagogía crítica artística no es una pedagogía para el arte, sino por el arte, alejada del arte como un objeto mercantilista y una formación instrumental de los estudiantes, como futuros eslabones de la cadena de producción en el mercado laboral (Escaño, 2009). Una pedagogía crítica artística desafía el imaginario popular proporcionando alternativas sociales y culturales, guiando pensamientos abstractos hacia la actuación, posibilitando universos no basados en el valor del trabajo como reconocimiento social (McLaren y Jaramillo, 2010). Por estos motivos, el potencial de las artes y la imaginación puede suponer un problema para aquellos que no buscan sino perpetuar el régimen social establecido. Las artes permiten mirar de otra forma, existiendo infinitas miradas al ser estas una construcción social y por tanto política. La mirada se construye partiendo del poder de la imaginación, no solo desempañando un proceso imaginativo sino como una fuente de contenidos, distorsionando la realidad (Eisner, 2002), habilitando opciones hasta entonces, invisibilizadas por el racionalismo impuesto y las grandes relatos modernistas. Un proceso tan sumamente valioso y potente, como lo es el ejercicio de la imaginación, parece ser prescindible en las escuelas cuando se despolitiza la educación, convirtiendo a las escuela en "zonas muertas de imaginación” (Giroux, 2015, p. 13)

\section{Los procesos intercreativos como estrategia docente}

Habiendo expuesto algunas de las posibilidades y problemáticas actuales más reseñables en relación a una educación (crítica) artística a continuación sintetizamos de manera breve una prácticas metodológicas reseñables en relación con las artes. Este "hacer" de la educación artística, se fundamenta en centrarse en el proceso y no en resultados predeterminados (Escaño y Marfil-Carmona, 2020; Mesias-Lema, 2019). El proceso es más importante que el resultado final creado por los estudiantes. La profesora y educadora artística Dipti Desai (2017), citando a Marit Dewhurst, nos habla de un proceso artístico que potencie maneras colectivas y críticas para comprometerse con la justicia social a través de las artes. Asumiendo la complejidad de la sociedad actual, efímera, líquida, es necesario implicar procesos artísticos que impulsen la reinterpretación, la remezcla frente a la acumulación pasiva de contenidos, haciendo más evidente la relación entre las esferas mediáticas y artísticas (Escaño y Marfil-Carmona, 2020). Las metodologías artísticas deben potenciar el análisis y reflexión, para una posterior acción, de nuestra sociedad postdigital sus atribuciones de remezcla y collage, dinámicas de entornos digitales que traspasando las barreras -cada vez más difusas- con entornos analógicos (Escaño, 2019). En esta realidad efímera y postdigital, es necesario una mirada transdisciplinar y no acogerse a determinadas estrategias o enfoques, los educadores artísticos deben permanecer atentos a los cambios sociales y juegos de poder sociales que se suceden para que puedan cuestionarse y replantearse su enseñanza (Desai, 2017).

Es necesario matizar, recogiendo los pensamientos de Efland et al., (2003), que cuando hablamos de una sociedad donde florecen manifestaciones artísticas como el collage y la 
remezcla y tratamos de aplicar estas consideraciones al currículum, no se trata de incluir información al azar o inconexa. Lo que realmente se atribuye en términos pedagógicos al collage y la remezcla es la construcción colectiva de significados entre profesores y alumnado. Así, el conocimiento se sucede mediante una red interconectada, no jerárquica donde pequeños relatos se suceden y se complementan. Un currículum intercultural y posmoderno, que introduce pedagogías críticas artísticas y aprovecha el factor intercreativo de la digitalidad, es sintomático del compromiso democrático y participativo, apostando por un cambio social.

La supervivencia del arte, comentaba Anderson (2003), reside en hacer comunidad, abarcando múltiples perspectivas culturales y narrativas. Las contrapedagogías online es un evento al que los educadores artísticos deben prestar atención (McLaren y Jaramillo, 2010). Todos los entornos digitales tienen en común el sentido de comunidad desde el que son concebidos, y son los propios participantes mediante estrategias colectivas, que dotan de sentido a los espacios digitales; interaccionando y creando un conocimiento común. Esta nueva potencia colectiva y online nos sitúa en un espacio proclive a la creatividad de la multitud, a la comunidad no presencial determinada por nuevos marcos biopolíticos (Zafra, 2015). Este hecho se ha materializado más explícitamente durante los meses de pandemia y confinamiento experimentados de manera globalizada. El papel de las artes y la cultura ha sido decisivo y transformativo con el objetivo de denunciar y servir de protesta social. Por tanto, como indican Freedman y Escaño (2020), analizar las prácticas artísticas durante la época de pandemia consecuencia del COVID-19, debe dirigirnos a otras prácticas educativas y artísticas desde perspectivas postdigitales que auguren por el cambio social desde creaciones en comunidad, desde una práctica intercreativa.

Siendo la intercreatividad, la noción más esclarecedora para definir la relación entre la cultura digital y la construcción del conocimiento resulta necesario profundizar en su naturaleza y examinarla como una estrategia pedagógica artística potencial que favorece el pensamiento crítico y la acción participativa y democrática. La intercreatividad como estrategia pedagógica para una educación capaz reflexiva y activa en aras del cambio social.

Este recurso favorecido por el advenimiento de las tecnologías de la comunicación y la información se define siguiendo a Tim Berners-Lee, (2000)de que deje de ser imparcial según el hardware o software que uses y que el usuario no lo sepa. De esta manera se fragmenta el web evitando que no sea universal y por tanto no sea práctico. $\operatorname{ln\backslash nCAPÍTULO:}$ TEJIENDO LA RED \n\nPag: 191, cita intercreatividad (último párrafo, como el hecho de crear en comunidad a través de dinámicas de interacción. El autor en su obra Tejiendo la Red analiza la importancia de la creación colectiva para avanzar como sociedad, es por ello por lo que las dinámicas de intercreación cobran importancia y resultan vitales para el desarrollo social. La intercreatividad no se circunscribe únicamente a un espacio digital, aunque su desarrollo es consecuentemente mayor en un terreno donde las posibilidades de interaccionar y las herramientas para la acción colectiva son ampliamente plurales y diversas.

La intercreatividad como proceso sociocultural es al mismo tiempo una experiencia de conocimiento colectivo. La noción de intercreatividad, al igual que la creatividad, 
se describe como un bien social -un bien común- y al igual que las manifestaciones artísticas y la cultura, la (inter)creatividad tiene su origen en lo social. Los seres humanos somos una especie colectiva por naturaleza, nos desarrollamos a través de innumerables interacciones con los demás a través de nuestros sentidos, siendo por tanto interacciones mediadas. El concepto de creatividad hoy día ha evolucionado desde una concepción platónica de mímesis de la naturaleza o aristotélica asemejando la creatividad con la locura y el caos (Jandrić, 2020). Las potencialidades de la intercreatividad son al mismo tiempo, subversivas y transformadoras. El término, no examinado en profundidad, se ha adherido a construcciones teóricas ya fundadas y con trayectoria en el ámbito académico y de la investigación como son inteligencia colectiva (Lévy, 2004) multitudes inteligentes (Rheingold, 2004) y creación colectiva (Casacuberta, 2003).

Sin embargo, el fundamento teórico sobre el que se asienta el concepto de intercreatividad es relativamente escaso a pesar de ser un recurso potencial dentro del marco educativo y, al mismo tiempo, claramente entrelazada con los actuales modos culturales y sociales de ver, ser y hacer. La intercreatividad es señalada por Escaño (2015) como una interdependencia en la creación, Camarero-Cano (2015)las personas se mueven en un entorno intercreativo, en el que somos capaces de co-crear de forma colaborativa permitiendo que la inteligencia colectiva de la sociedad fluya.InComo veremos, las nuevas tecnologías digitales han transformado la forma en que los seres humanos se comunican y, por lo tanto, el desarrollo de la creatividad también ha experimentado un cambio. Esto ha dado lugar a la intercreatividad, la interacción de las personas dentro de un espacio analógico-digital que ha hecho posible la co-creación a gran escala. Gracias a la suma del conocimiento individual hemos logrado la inteligencia colectiva. In $\backslash n$ Autores analizan intercreatividad: Lucia Camarero (2014 destaca su carácter social y de intercambio creativo y su creador y Berners-Lee (2000) argumenta que las conexiones correctas entre personas no tienen tanto que ver con el tamaño del grupo sino con los (no) límites, potenciados por la postdigitalidad.

En particular, la intercreatividad reúne dos significados relevantes estrechamente relacionados con el campo transformador de la pedagogía crítica: la interacción y la creación. En consecuencia -pasando de un enfoque tradicional- el principal desafío educativo es permitir que los individuos transformen libremente su realidad a través de ejercicios de reflexión y crítica previamente promovidos por la interacción inherente al ser humano. En relación con este contexto educativo, a través de la intercreatividad los estudiantes tejen una red en la que colaboran, intercambian, crean o modifican información, convirtiéndola en un conocimiento cada vez más complejo.

Como beneficio de la intercreatividad en relación con la educación, podríamos añadir que cuando enfatizamos el proceso en detrimento del resultado final, abogamos por la interacción como herramienta de aprendizaje para que la creatividad sea altamente estimulada. El aprendizaje parte de una reflexión focalizada en una acción posterior, de manera que se realiza un pensamiento para luego llevar a cabo una transformación totalmente autogestionada. Como señala Mihaly Ciskszentmihalyi (1998), la creatividad es un proceso sistémico, no es individual. No se desarrolla únicamente en la cabeza de los seres humanos, sino en la interacción que se produce entre las personas, sus pensamientos 
y el ambiente sociocultural en el que todos y cada uno de nosotros estamos inmersos. Este pensamiento es extrapolable a nuestro concepto intercreativo ya que de manera indirecta alude a él.

\section{Conclusiones para seguir avanzando}

Podríamos concluir la presente reflexión apuntando algunas nociones que nos permitan establecer relaciones entre los procesos intercreativos y su práctica. La intercreatividad, como hecho educativo, nos recuerda a lo que Freire denominó la teoría dialógica, una teoría que parte de la acción debido a que los sujetos se encuentran unos con otros para transformar el mundo de una manera conjunta, colaborando sin atisbo de dominación (Freire, 1968). Siendo la intercreatividad un encuentro entre iguales con el propósito de interactuar para resolver problemas juntos, podríamos intuir el mismo interés transformador citado por Freire en la teoría antes expuesta. La noción creadora que nos aporta el ejercicio intercreativo no solo está relacionado con el hecho de inventar y dejar surgir algo novedoso sin constancia de precedentes. Si creamos a partir de algo existente, modificándolo y reinventándolo puede ser considerado incluso un hecho más creativo que la fuente original en cuestión (Lessig, 2012). Si entendemos la educación como una herramienta de transformación social a través de interacciones sociales y entendiendo la creatividad como un instrumento para esa misma transformación, el término intercreatividad cobra mayor importancia.

La intercreatividad o - lo que es lo mismo - la interacción, sociabilización o relaciones entre los iguales con el objetivo de colaborar o crear de manera conjunta, es una de las claves para entender el aprendizaje como un proceso constante y reflexivo. Hoy día cobra si cabe más importancia cuando nuestra contemporaneidad se encuentra progresivamente más basada en un sistema informacional. El trabajo inmaterial, a través de ideas o información intangible, es una realidad y la intercreatividad es crucial en términos sociales (Jandrić, 2020). Dentro del proceso intercreativo, toda voz es esencial ya que la suma de todas ellas da lugar a una voz global y colectiva, que no tendría lugar si no existiera una diferencia y una diversidad. Nuestra identidad se configura a través de las relaciones sociales y culturales, por tanto, existe una interdependencia latente. Los ejercicios de creación grupal, potenciados a través de actividades artísticas son mecanismos eficaces para tomar conciencia de estos procesos de interdependencia a través de prácticas intercreativas (Escaño, 2015). Por este motivo, pensamos que la creatividad debe ser valorada como un transcurso de acontecimientos y vaivenes que incide en nosotros mismos y nuestro entorno. Dicho esto, resulta imposible que se considere un hecho estático, un acontecimiento en un momento determinado, ya que es el resultado de un conjunto de interacciones cognitivas, personales y situacionales, y ninguna de estas tres características es en su esencia estática o atemporal. Los procesos creativos — como señala Mesías-Lema (2019)— son caóticos e interconectados, los alumnos se nutren del proceso creativos valorándolo más que el resultado final.

La colaboración y la creación son conceptos básicos a la hora de hablar de educación y cambio social. Educar en creatividad es educar en reflexión, autonomía y cambio, en 
consciencia crítica y transformación social es educar, en consecuencia, por y para una mejora personal y social. Un discurso que se aproxima a la práctica intercreativa desde una concepción educativa crítica, reconoce la concepción artística como un poderoso recurso pedagógico en el aula. La intercreatividad se erige como una práctica artística y una estrategia educativa a través de la creación colectiva que focaliza su atención en la mejora y el progreso social. Reconocer este hecho es ensalzar el valor de la educación artística como un importante recurso crítico y transformativo dentro del seno educativo y de la cultura y la sociedad que nos atraviesa.

\section{Referencias bibliográficas}

Anderson, T. (2003). Art education for life. International Journal of Art and Design Education, 22(1), 58-66. https://doi.org/10.1111/1468-5949.00339.

AprendemosJuntos. (1 de abril de 2020). Es el momento de enseñar a los niños a entender el mundo. Noam Chomsky, lingüista y profesor. [Archivo de video] YouTube. https://www. youtube.com $/$ watch? $\mathrm{v}=$ OEdfJP9S4NE\& feature $=\mathrm{emb}$ title.

Arañó, J. C. (2003). La investigación en educación artística, entre la utopía y la irrealidad. Educación Artística: Revista de Investigación (EARI), 1, 159-164.

Barbosa, A. M. (2012). 1ª Bienal de Educación Artística "Geografía de un vínculo". El Arte/Educación Que Necesitamos, 25-33. Maldonado.

Barragán, J. M. (2005). Educación artística: perspectivas críticas y práctica educativa. En Investigación en educación artística: temas, métodos y técnicas de indagación sobre el aprendizaje y la enseñanza de las artes y culturas visuales (pp. 43-81). Universidad de Granada.

Bauman, Z. (2006). Vida líquida. Paidós.

Berners-Lee, T. (2000). Tejiendo la red. Siglo XXI de España.

Camarero-Cano, L. (2015). Conectividad e intercreatividad en las comunidades tecnosociales un estudio de caso: la liga de optimistas pragmáticos. [Tesis doctoral, UNED - Universidad Nacional de Educación a Distancia. http://e-spacio.uned.es/fez/view/ tesisuned:Educacion-Lcamarero.

Casacuberta, D. (2003). Creación colectiva. En Internet el creador es el público. Gedisa.

Chalmers, F. G. (2003). Arte, educación y diversidad cultural. Paidós.

Ciskszentmihalyi, M. (1998). Creatividad. El fluir y la psicología del descubrimiento y la invención. Paidós.

Desai, D. (2017). Artistic activism in dangerous times: Teaching and learning against the 
grain. Visual Inquiry: Learning and Teaching Art, 6(2), 135-145. https://doi.org/10.1386/ vi.6.2.135_2.

Dewey, J. (2008). El arte como experiencia. Paidós.

Efland, A. D., Freedman, K., y Stuhr, P. (2003). La educación en el arte posmoderno. Paidós.

Eisner, E. W. (2002). Qué enseñan las artes y en qué se nota. En El arte y la creación de la mente (pp. 97-123). Paidós.

Escaño, C. (2009). Hipercontextos, Arte y Educación. IN-Visibilidades, 29-39.

Escaño, C. (2015). Por una Educación Hacker: Valores Educativos del Arte de Internet Enmarcado en la Cultura Libre. IN-Visibilidades, 8, 21-35.

Escaño, C. (2019). Lo que no se ve no existe. Educación y transformación social a través de la imagen. En Lo que no se ve no existe. Artes, imagen y educación para el desarrollo. (pp. 181-198). UNED - Universidad Nacional de Educación a Distancia.

Escaño, C., y Marfil-Carmona, R. (2020). Artes y su educación: metodologías didácticas artísticas para la innovación y el cambio social. En Aprendizaje más allá de las aulas. Didácticas especificas en contextos no formales. (pp. 94-119). Humanidades.

Freedman, K., y Escaño, C. (2020). Reflections IX and X: Reflections from Education and the Arts in the COVID-19 Era. Communiars. Revista de Imagen, Artes y Educacion Crítica y Social, 4, 25-28. https://doi.org/10.1017/CBO9781107415324.004.

Freire, P. (1968). Pedagogía del oprimido. Siglo XXI.

Giroux, H. (2015). Cuando las escuelas se convierten en zonas muertas de la imaginación: manifiesto de la pedagogía crítica. Revista de Educación, 8, 11-26.

Jandrić, P. (2020). Creativity and Collective Intelligence. En M. A. Peters y R. Heraud

(Eds.), Encyclopedia of Educational Innovation (pp. 1-5). https://doi.org/10.1007/978981-13-2262-4_65-1.

Jandrić, P., et al. (2020). Teaching in the Age of Covid-19. Postdigital Science and Education, 2, 1069-1230. https://doi.org/10.1007/s42438-020-00169-6.

Jandrić, P., Knox, J., Besley, T., Ryberg, T., Suoranta, J., y Hayes, S. (2018). Postdigital science and education. Educational Philosophy and Theory, 50(10), 893-899. https://doi. org/10.1080/00131857.2018.1454000.

Jimenez, R. (2020). Un docente revolucionario para la formación artística (decolonial) contemporánea. Educación Artística: Revista de Investigación (EARI), 11, 114-138. http:// dx.doi.org/10.7203/eari.11.15237.

Ley Orgánica 3/2020, de 29 de diciembre, por la que se modifica la Ley Orgánica 2/2006, 
de 3 de mayo, de Educación. Boletín Oficial del Estado, 340, de 30 de diciembre de 2020, 122868 a 122953. https://www.boe.es/boe/dias/2020/12/30/pdfs/BOE-A-2020-17264.pdf.

Lessig, L. (2012). Remix. Cultura de la remezcla y derechos de autor en el entorno digital. Icaria.

Lévy, P. (2004). Inteligencia colectiva. Por una antropología del ciberespacio. http:// inteligenciacolectiva.bvsalud.org/public/documents/pdf/es/inteligenciaColectiva.pdf.

López Fernández-Cao, M. (2008). Cognición y emoción: el derecho a la experiencia a través del arte. Pulso: Revista de Educación, 31, 221-232.

Maeso-Broncano, A. (2017). La dimensión político-social del arte de acción y su incidencia en los entornos pedagógicos. Enfoque y prácticas performáticas como recurso educativo. [Tesis doctoral, Universidad de Sevilla]. https://idus.us.es/handle/11441/77298.

Markus, G. (2020). El virus, el sistema letal y algunas pistas para después de la pandemia. En P. Amadeo (Ed.), Sopa de Wuhan (pp. 129-135). ASPO (Aislamiento Social Preventivo y Obligatorio).

McLaren, P., y Jaramillo, N. (2010). Las artes, la estética y la pedagogía crítica. En R. Gutiérrez y C. Escaño (Eds.), III Congreso Internacional de Educación Artística y Visual: pensamiento crítico y globalización. (pp. 37-60). Servicio de Publicaciones y Divulgación Científica de la Universidad de Málaga.

Mesias-Lema, J. M. (2019). Educación artística sensible. Cartografia contemporánea para arteducadores. Graó.

Palacios-Garrido, A. (1999). Educación artística y cultural: un proyecto de didáctica de la expresión plástica en la formación inicial del profesorado. Arte, Individuo y Sociedad, 11, 147-162. https://doi.org/10.5209/ARIS.6796.

Read, H. (1969). Función de las artes en la sociedad contemporánea. En Arte y alienación. Proyección.

Rheingold, H. (2004). Las multitudes inteligentes: el poder de las multitudes móviles. En Multitudes inteligentes. Gedisa.

Robinson, K. (2009). El elemento. Grijalbo.

Vaquero, C., y Gómez del Águila, L. M. (2018). Educación artística, desconsideración social y falta de expectativas. Consecuencias de la reproducción como paradigma noeducativo. Educación Artística: Revista de Investigación (EARI), 9, 220-236. https://doi. org/10.7203/eari.9.12051.

Zafra, R. (2015). Educación y cultura-red: potencias y contradicciones para una transformación necesaria. Profesorado: Revista de Curriculum y Formación Del Profesorado, 19(2), 11-24. 